\title{
Social buffering and contact transmission: Network connections have beneficial and detrimental effects on Shigella infection risk among captive rhesus macaques
}

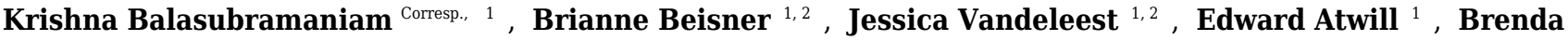
McCowan ${ }^{1,2}$

${ }^{1}$ Department of Population Health \& Reproduction, School of Veterinary Medicine, University of California, Davis, Davis, California, United States

2 Brain, Mind \& Behavior, California National Primate Research Center, University of California, Davis, Davis, California, United States
\end{abstract}

Corresponding Author: Krishna Balasubramaniam

Email address: krishnanatarajan@ucdavis.edu

In social animals, group living may impact the risk of infectious disease acquisition in two ways. On the one hand, social connectedness puts individuals at greater risk or susceptibility for acquiring enteric pathogens via contact-mediated transmission. Yet conversely, in strongly bonded societies like humans and some nonhuman primates, having close connections and strong social ties of support can also socially buffer individuals against susceptibility or transmissibility of infectious agents. Using social network analyses, we assessed the potentially competing roles of contact-mediated transmission and social buffering on the risk of infection from an enteric bacterial pathogen (Shigella flexneri) among captive groups of rhesus macaques (Macaca mulatta). Our results indicate that, within two macaque groups, individuals possessing more direct and especially indirect connections in their grooming and huddling social networks were less susceptible to infection. These results are in sharp contrast to several previous studies that indicate that increased (direct) contact-mediated transmission facilitates infectious disease transmission, including our own findings in a third macaque group in which individuals central in their huddling network and/or which initiated more fights were more likely to be infected. In summary, our findings reveal that an individual's social connections may increase or decrease its chances of acquiring infectious agents. They extend the applicability of the social buffering hypothesis, beyond just stress and immunefunction-related health benefits, to the additional health outcome of infectious disease resistance. Finally, we speculate that the circumstances under which social buffering versus contact-mediated transmission may occur could depend on multiple factors, such as living condition, pathogen-specific transmission routes, and/or an overall social context such as a group's social stability. 
1 Social buffering and contact transmission: Network connections have beneficial and

2 detrimental effects on Shigella infection risk among captive rhesus macaques

3

4 Krishna N. Balasubramaniam ${ }^{\text {a }}$ Ph.D.

5 Brianne A. Beisner ${ }^{\mathrm{a}, \mathrm{b}}$ Ph.D.

6 Jessica Vandeleest ${ }^{\mathrm{a}, \mathrm{b}} \mathrm{Ph} . \mathrm{D}$.

$7 \quad$ Rob Atwilla D.V.M., Ph.D.

8 Brenda McCowan ${ }^{\mathrm{a}, \mathrm{b}}$ Ph.D.

9

10 aDepartment of Population Health \& Reproduction, School of Veterinary Medicine, University

11 of California, Davis, Davis, California, United States;

12 brain, Mind \& Behavior, California National Primate Research Center, University of California, 13 Davis, Davis, California, United States;

14

15

16

Corresponding author:

Krishna N. Balasubramaniam

One Shields Avenue 


\section{Abstract}

In social animals, group living may impact the risk of infectious disease acquisition in two ways. On the one hand, social connectedness puts individuals at greater risk or susceptibility for acquiring enteric pathogens via contact-mediated transmission. Yet conversely, in strongly bonded societies like humans and some nonhuman primates, having close connections and strong social ties of support can also socially buffer individuals against susceptibility or transmissibility of infectious agents. Using social network analyses, we assessed the potentially competing roles of contact-mediated transmission and social buffering on the risk of infection from an enteric bacterial pathogen (Shigella flexneri) among captive groups of rhesus macaques (Macaca mulatta). Our results indicate that, within two macaque groups, individuals possessing more direct and especially indirect connections in their grooming and huddling social networks were less susceptible to infection. These results are in sharp contrast to several previous studies that indicate that increased (direct) contact-mediated transmission facilitates infectious disease transmission, including our own findings in a third macaque group in which individuals central in their huddling network and/or which initiated more fights were more likely to be infected. In summary, our findings reveal that an individual's social connections may increase or decrease its chances of acquiring infectious agents. They extend the applicability of the social buffering hypothesis, beyond just stress and immune-function-related health benefits, to the additional health outcome of infectious disease resistance. Finally, we speculate that the circumstances under which social buffering versus contact-mediated transmission may occur could depend on multiple factors, such as living condition, pathogen-specific transmission routes, and/or an overall social context such as a group's social stability. 


\section{Introduction}

52 In humans and other animals, the strength and diversity of social relationships strongly

53 influence the risk of acquiring infectious diseases (Alexander, 1974; Drewe \& Perkins, 2015;

54 McCowan et al., 2016; Nunn, 2012). In addition to life-history traits of the host, biology of the

55 pathogen, and the degree of contact with contaminated environmental sources (Drewe \& Perkins,

56 2015; Kappeler et al., 2015), social connections with group conspecifics may also influence the

57 risk of acquiring and/or transmitting a pathogen (Drewe \& Perkins, 2015). This may occur in two

58 ways. On the one hand, increased connections may lead to greater chances of infection from

59 pathogens via contact-mediated transmission (Drewe \& Perkins, 2015), making infectious

60 disease acquisition a major cost of social living (Alexander, 1974; Freeland, 1976; Loehle, 1995;

61 MacIntosh et al., 2012). Yet social connections may also mitigate the impact of stressors or

62 immunosuppressive effects of stress, thereby socially buffering an individual to decrease their

63 susceptibility to infection (Hennessy et al., 2009; Kikusui et al., 2006; McCowan et al., 2016;

64 Sapolsky, 2005; Sapolsky et al., 2000; Segerstrom \& Miller, 2004; Young et al., 2014). To better

65 understand the impact of social life on disease risk, it is necessary to characterize the potentially

66 competing impacts of both greater contact-mediated transmission and social buffering on

67 susceptibility to, and transmission of pathogens.

68 Traditional models of disease acquisition and transmission in social systems assume that

69 individuals interact randomly (Anderson \& May, 1992). In reality, individuals differ in how they

70 navigate their social environment, which reflects differences in their social strategies to

71 maximize fitness (Alexander, 1974; Bansal et al., 2007). Such heterogeneity can be modeled

72 using social network analysis (Krause et al., 2007; McCowan et al., 2008). Most recent work

73 implementing social network analysis to investigate infectious agent epidemiology in animal 
74 systems has focused on contact-mediated infection and transmission (reviewed in (Drewe \&

75 Perkins, 2015)). Broadly, this phenomenon predicts that greater social connectedness, via more

76 frequent contact rates with infected individuals within one's social network, increases one's risk

77 of being infected by socially-transmitted pathogens (Drewe \& Perkins, 2015). Specifically, being

78 very central, and/or showing increased rates of contact-associated behaviors (e.g. social or

79 allogrooming) within a social network has been shown to impact a variety of infectious-disease

80 associated health outcomes, such as increasing endoparasite load, prevalence of a specific

81 pathogen, and pathogenic diversity of individuals (Nunn, 2012). Indeed, contact-mediated

82 transmission of pathogens has been shown to occur among a variety of animal social groups,

83 such as group-living lizards (Godfrey et al., 2009), Tasmanian devils (Hamede et al., 2009),

84 Belding's ground-squirrels (VanderWaal et al., 2013), and nonhuman primates (MacIntosh et al.,

85 2012; Rimbach et al., 2015). Furthermore, epidemiologists speculate that highly central

86 individuals may also act as superspreaders of pathogens because they can also transmit an

87 infection to their neighbors (Drewe \& Perkins, 2015; Lloyd-Smith et al., 2005). Yet an

88 individual's susceptibility versus resistance to infection may depend not just on its direct

89 connections but also on its neighbors' connections (Farine \& Whitehead, 2015). To date, the

90 impact of such indirect or secondary connections on infection risk has received relatively less

91 attention in epidemiological studies (but see (MacIntosh et al., 2012)).

92 Even less explored is the possibility that having more direct or indirect connections in a

93 network may, via social buffering, protect individuals from social and environmental stressors

94 (Kaplan et al., 1991; Young et al., 2014), and decrease rather than increase their susceptibilities

95 to infection. In societies where individuals maintain strong social bonds (e.g. humans, some

96 nonhuman primates like baboons (Papio sp.) and macaques (Macaca sp.)), social buffering is 
97 well-documented - having more social ties can mitigate stress to generate positive health

98 outcomes (Cobb, 1976; Young et al., 2014) and indeed, enhance longevity and survival (Archie

99 et al., 2014; Silk et al., 2010). In humans for example, having more connections who provide

100 social support during times of conflict alleviates stress-levels (Janowski et al., 2012) and

101 decreases susceptibility to non-communicable diseases such as cancer and cardiovascular disease

102 (Uchino, 2004, 2009). In nonhuman primates, grooming (an affiliative social interaction) may be

103 exchanged among group members for access to social support (Seyfarth, 1977), and is also

104 known to lower circulating levels of glucocorticoids by reducing activation of the hypothalamic-

105 pituitary-adrenal (HPA) axis (Young et al., 2014). Socio-positive interactions appear to be key in

106 alleviating stress, which both enhances immune function (Sapolsky et al., 2000) and decreases

107 susceptibility to infectious agents (Cohen et al., 2007; Segerstrom \& Miller, 2004). Yet to our

108 knowledge, no work has investigated the effects of social buffering on health using network-

109 based analytical approaches, or in the context of infectious diseases. To better understand how

110 social connections impact disease-related health outcomes, it is imperative to assess the effects of

111 contact-mediated transmission and social buffering, particularly indirect social connections

112 through which group members may elicit social support, on the risk of acquiring infectious 113 agents.

114 Here we use a nonhuman primate model, the rhesus macaque (Macaca mulatta), to test 115 whether the risk of infection from a bacterial pathogen (Shigella flexneri) is higher in socially

116 well-connected individuals or lower in individuals buffered by having access to social support.

117 Enteric bacterial pathogens, such as Shigella, are ubiquitous, well documented, and among the

118 most communicable pathogens in humans (DuPont, 2000). Although infected individuals can

119 occasionally remain asymptomatic, doses as low as between 20-200 organisms may be sufficient 
120 to cause symptoms of Shigellosis, ranging from acute to severe diarrhea, fever, and even death

121 (DuPont, 2000). Shigella transmission in humans occurs via the fecal-oral route, or person-to-

122 person contact, as well as consumption of contaminated food and water sources (Anderson \&

123 May, 1992; Scallan et al., 2011). Among nonhuman primates, Shigella (and indeed other enteric

124 bacteria) are surprisingly understudied in comparison with other pathogens

125 (www.mammalparasites.org) (Nunn \& Altizer, 2006). The few studies to date report low

126 prevalence in some free-living populations that live in close proximity to humans (e.g. rhesus

127 macaques: (Beisner et al., 2016); Savannah baboons: (Drewe et al., 2012; Harper et al., 2012)).

128 Although the dosage levels of Shigella flexneri that may generate virulent effects among

129 primates is unclear, the occurrence of acute enteritis in rhesus macaques housed in captivity has

130 been linked to infection this pathogen (Lee et al., 2011). There have also been two documented

131 outbreaks of Shigellosis among a semi-free ranging rhesus macaques in Puerto Rico, resulting in

132 enteritis, abortions, and increased mortality rates among pregnant monkeys (Kessler \& Rawlins,

133 2016). Such documentations of virulent infection among primates, further to its well-established

134 social- and/or environmental-contact mediated transmission routes among humans, make

135 Shigella an ideal pathogen for investigating the effects of social network connectedness on

136 susceptibility versus resistance to infection risk.

137 Rhesus macaques are biologically, socially and cognitively analogous to human societies

138 (Cobb, 1976; Suomi, 2011). They live in large ( 20-150 individuals), multi-male-multi-female

139 social groups (Thierry, 2007). Individuals maintain and reinforce their social relationships using

140 a variety of behaviors, such as aggression, grooming, and social huddling (Lindburg, 1971; Sade,

141 1972). These behaviors are heterogeneously distributed in accordance with sex, kinship, age-sex

142 category, and dominance ranks of individuals (Thierry, 2007). For example, social bonds 
143 established via grooming tend to be strongest among closely related females, and/or females with

144 adjacent dominance rank which is also inherited in accordance with kinship (e.g. (Berman \&

145 Thierry, 2010; Chapais, 2006; Sade, 1972)). From a health perspective, these behaviors typically

146 bring individuals into physical contact and can also influence levels of social tension or stress.

147 For example, grooming has well-documented fitness, particularly stress-relieving, benefits in

148 nonhuman primates (summarized in (Henzi \& Barrett, 1999)) as well as in other animal taxa (e.g.

149 social insects: (Moore et al., 1995) horses: (Kimura, 1998)). Similarly, although the functional

150 significance of huddling among macaques is yet to be documented, this behavior may be

151 considered to be physiologically (involving relaxed body-to-body contact) analogous to hugging

152 among humans, which has been shown to socially buffer individuals from infection by the

153 common-cold virus (Cohen et al., 2015). Conversely, grooming can also serve as a contact-route

154 of infection (Japanese macaques (M. fuscata: (MacIntosh et al., 2012); other primates such as

155 brown spider monkeys (Ateles hybridus: (Rimbach et al., 2015)). Aggressive interactions have

156 the potential to enhance susceptibility to infection by bringing aggressors and recipients into

157 physical contact (Drewe, 2010a), or by elevating stress-levels in recipients of aggression (e.g.

158 (Muller \& Wrangham, 2004)). Among Japanese macaques (MacIntosh et al., 2012) revealed that

159 dominance rank, an outcome of dyadic aggressive interactions, positively affected parasite

160 diversity but not fecal glucocorticoids, indicating a contact- rather than a stress-mediated

161 infection-route. In primate species like rhesus macaques that are characterized by despotic social

162 relationships, the effect of dominance rank on infection risk may be somewhat redundant to

163 examining those of social network connectedness, since social rank directly effects such

164 connectedness and centrality (e.g. (MacIntosh et al., 2012; Sueur et al., 2011b)). Yet other than

165 (MacIntosh et al., 2012), we are unaware of any work that has simultaneously examined the 
166 effect(s) of greater network connectedness (which may occur via dominance rank) to specifically

167 evaluate the contrasting roles of contact-mediated transmission and social buffering, on

168 infectious disease risk.

169 We first establish (1) the prevalence of Shigella flexneri within each of three groups of

170 captive rhesus macaques. For each of these groups, we next reconstruct each of three types of

171 social networks based on grooming, huddling, and aggressive interactions respectively. Upon

172 doing so, we investigated whether (2) greater centrality and/or social connectedness through both

173 direct and (where relevant) indirect or secondary connections in these networks mitigated

174 macaques' risk of infection via social buffering, versus enhanced this risk through contact-

175 mediated transmission. Specifically, if connections socially buffer individuals against Shigella

176 infection risk, we predicted that this bacterial pathogen will be the least prevalent among

177 individuals with the most direct and/or indirect social connections. On the other hand, if

178 possessing connections enhance infection risk via contact-mediated transmission, we predicted

179 that infection will be most prevalent among individuals with the highest number and/or diversity

180 of direct connections. Finally, we also ascertained whether (3) attributes of individuals other than

181 their network positions, but which may influence their positions (particularly sex, dominance

182 rank, and the certainty of their dominance relationships ((Fujii et al., 2014); see Methods)), also

183 influenced their risk of infection. Specifically, we asked whether Shigella infection was least

184 likely among high-ranking females who typically form the strongest social bonds. Further, given

185 that increased uncertainty in dominance relationships could be more stressful (Vandeleest et al.,

186 2016), we asked whether the effect of possessing increased connections on socially buffering

187 individuals from infection risk was more clearly discernible among individuals who also had

188 more uncertain compared to certain dominance relationships. 
190

191

192

193

194

195

196

197

198

199

200

201

202

203

204

205

206

207

208

209

210

211

Materials and Methods

Study location and subjects: The study was conducted at the California National Primate Research Center (CNPRC) and the School of Veterinary Medicine (SVM), University of California at Davis. The subjects were 299 adult rhesus macaques (90 males, 209 females) between the ages of 3 and 29 (mean $=7.7$ years), distributed across three social groups ( 101 in Group I, 96 in Group II, and 102 in Group III). The groups were housed in 0.2 ha outdoor enclosures containing multiple A-frame structures, suspended barrels, swings, and perches; they were free to engage in social interaction. Animals were fed a standard diet of monkey chow twice per day at approximately $0700 \mathrm{~h}$ and between 1430 and $1530 \mathrm{~h}$. Fresh fruit or vegetables were provided one time per week and seed mixture provided daily. Water was available ad libitum. The outdoor housing facilities are exposed to a minimum level of disturbance, and may be considered semi-naturalistic. The protocols used for this research were approved by the UC Davis Institutional Animal Care and Use Committee (IACUC), and were in accordance with the legal requirements of the jurisdictions in which the research was conducted.

Behavioral Data Collection: Behavioral data were collected on each group for 6 weeks, two groups in the spring (Group I: March-April 2013; Group III: March-April 2014) and one in the fall (Group II: September-October 2014). For each group, three observers collected data for 6 hours on 4 days per week from 0900-1200 h and 1300-1600 h, using (i) an Event Sampling design for aggressive interactions, and (ii) Scan Sampling for affiliative grooming and huddling interactions (Altmann, 1974). These have been shown to optimize reliable collection of data in large groups of subjects (McCowan et al., 2011), both improving statistical power and circumventing nonindependence issues that may affect the computation of reliable social 
212 network metrics (Farine \& Whitehead, 2015). For each 'event', the identities of the initiator and

213 recipient macaque, and their specific behavior(s) were recorded. Grooming was defined as an

214 animal cleaning or manipulating the fur of another individual. Huddling was defined as the

215 occurrence of all forms of body-contact, including (but not restricted to) ventral contact or an

216 embrace between two individuals that did not involve a social behavioral interaction (e.g.

217 grooming, contact aggression). Aggression was categorized according to severity and included

218 threat (open mouth stare, brow flash, ear flap), mild aggression (threat and follow, lunge, push,

219 slap, chase $<6$ meters), moderate aggression (grapple, wrestle, chase $>6$ meters), and intense

220 aggression (pin or bite). We recorded events of polyadic aggression as a series of dyadic

221 interactions. Data on all dyadic and polyadic interactions were used to calculate the network

222 metrics of degree and strength. Submission categories included freeze/turn away, move away,

223 run away $<6$ meters, run away $>6$ meters, and crouch. For computing dominance rank and

224 certainty (see below), we used only data on dyadic aggressive and submissive interactions.

225 Social Network and Dominance Metrics: For each of the three groups, we constructed

226 three types of social networks: (1) groom, (2) huddle, and (3) aggression. From undirected

227 grooming and huddling networks, we calculated the number of direct connections (or degree

228 centrality), the total number of shortest pathways between other pairs of animals that pass

229 through an individual (or betweenness centrality), and a metric of social capital that takes both

230 direct and indirect connections of individuals into consideration (eigenvector centrality) (Farine

231 \& Whitehead, 2015; Makagon et al., 2012). For directed networks of grooming and aggression,

232 we also calculated the number of connections given and received (in- and out-degree

233 respectively), and the strengths of these connections weighted by the frequencies of interactions

234 (in- and out-strength respectively). These were computed using the Statnet and Sna (Handcock et 
235 al., 2006) packages in R. Given its estimation of an individual's direct connections, degree

236 centrality has been extensively used in epidemiological studies to date that have focused on

237 contact-mediated pathogen transmission (Drewe, 2010a; Drewe \& Perkins, 2015; Rimbach et al.,

238 2015). On the other hand, in- and out-degree and strength in grooming may also be indicative of

239 reduced infection risk via social buffering, given that grooming interactions have well-

240 documented benefits of lowering the physiological stress levels among both givers and receivers

241 (Henzi \& Barrett, 1999; Smutt et al., 2007; Young et al., 2014). Although betweenness centrality

242 has been less commonly used in epidemiological studies, it has been proposed as being a key

243 metric in predicting the potential for the flow of infectious agents through the generally more

244 densely connected social networks (Drewe \& Perkins, 2015; Farine \& Whitehead, 2015;

245 Newman, 2005; VanderWaal et al., 2014). Finally, as an indicator of access to social support via

246 indexing secondary connections, eigenvector centrality may be particularly key in determining

247 whether the possession of such extended support circles beneficially inhibits (via buffering),

248 versus detrimentally exposes an individual to infection risk (via contact-transmission: e.g.

249 (MacIntosh et al., 2012)).

250 We implemented a recently developed network algorithm, Percolation-and-Conductance

251 (or Perc: (Fujii et al., 2014)), to compute dominance ranks and certainties from aggression

252 networks constructed from dyadic interactions only. This method combines information from

253 direct dominance interactions with information from multiple indirect dominance pathways (via

254 common third parties) to quantify dyadic dominance relationships (Fujii et al., 2014). This

255 algorithm identifies all potential flow pathways in the network, weighting the contribution of

256 each path to the imputed matrix by its likelihood of being traversed by the random walk. This

257 method yields two outputs: a matrix of dyadic dominance certainty values (range: $0-1$ ) and the 
258 lowest-cost linear rank order. The former represent the cumulative information from all network

259 pathways between each pair of animals. A dominance certainty value of 1 reflects the highest

260 possible certainty that the row animal outranks the column animal (and 0 reflects the highest

261 possible certainty that the column animal outranks the row animal) whereas 0.5 means the

262 dominance relationship is perfectly ambiguous. To compute the average dominance certainty of

263 each individual, we transformed all dyadic dominance certainty values between 0.5 and 1 , and

264 calculated the row-wise average for each animal. We also transformed ordinal dominance ranks

265 for each group into the proportion of animals outranked within their respective groups (i.e. 0 is

266 the lowest ranked animal and 1 is the highest ranked animal). This measure of 'percentile ranks'

267 was used in the place of ordinal ranks in the analyses.

268 Pathogen Characterization: Prior to fecal collection, animals were immobilized (10

$269 \mathrm{mg} / \mathrm{kg}$ of ketamine) and given standard physical examinations by veterinary staff (e.g., checked

270 for injuries, weighed). We collected two fresh fecal swabs from every macaque at the end of the

271 behavioral observation period following previously published methods (Good et al., 1969).

272 Briefly, a sterile cotton-tip swab was inserted into the rectum of each individual, rotated gently to

273 collect fecal material, and immediately immersed into a $15 \mathrm{ml}$ test-tube (labeled with the animal

274 ID) containing sterile Tryptic Soy Broth (TSB; BD Franklin Lakes, NJ, USA); a duplicate

275 sample was taken using another sterile swab and placed into a second TSB tube. The samples

276 were incubated within 4 hours of collection; the tubes were incubated with orbital rotation of 100

277 rpm at (1) $25^{\circ} \mathrm{C}$ for $2 \mathrm{~h},(2) 42^{\circ} \mathrm{C}$ for $8 \mathrm{~h}$, and (3) held static at $6^{\circ} \mathrm{C}$ overnight. Shigella was

278 isolated from the TSB enrichment; $\sim 10 \mu \mathrm{L}$ enrichment was struck for isolation onto MacConkey

279 agar plates (BD Franklin Lakes, NJ USA), and incubated at $37^{\circ} \mathrm{C}$ for $18-24$ hours. Suspect

280 colonies were further isolated and characterized on Xylose Lysine Deoxycholate agar plates 
281 (XLD, BD Franklin Lakes, NJ USA). Two isolated colonies per sample were biochemically

282 confirmed using (i) Triple Sugar Iron (TSI) (Remel Lenexa, KS), (ii) Citrate (Remel Lenexa, KS

283 USA), (iii) Urea (BD Franklin Lakes, NJ USA), and (iv) the Methyl Red - Voges-Proskauer

284 (MR-VP) test (BD Franklin Lakes). Individuals which tested positive from at least one of the two

285 swabs were categorized as infected, with those that tested negative from both swabs deemed

286 uninfected.

287 Data analysis: The low prevalence of bacterial infection among macaques (e.g. (Beisner

288 et al., 2016; Drewe et al., 2012); see Results) has the potential to impact the statistical power of

289 our analyses. We therefore conducted some diagnostic assessments to determine whether there

290 was sufficient homogeneity of the data across groups to warrant combining them in a single

291 model-set. Diagnostic plots (Figure 1) showed that while the patterns of relationships between

292 Shigella infection and network metrics appeared more similar for Groups I and II, those for

293 Group III were starkly different. These are further supported by scatter-plots that showed similar

294 infection patterns (concentrated among individuals moderately or least central in their grooming

295 and huddling networks) in Groups I and II, but a different pattern of infection being widely

296 distributed across individuals of varying centralities in Group III (Supporting Figure 1). Finally,

297 Groups I and II were highly similar, and different from Group III, in the age structure of

298 individuals, time of formation, and the number of matrilines, any or all of which may impact

299 heterogeneity in social network dynamics (Supporting Table 1; see Discussion). For these

300 reasons, we chose to combine Groups I and II into a single, homogeneous population for our

301 analyses, and analyzed Group III as a second, separate dataset.

302

To investigate our aims, we implemented an Information-Theoretical approach to

303 construct generalized linear mixed-effects models (Burnham et al., 2011; Grueber et al., 2011; 
304 Whittingham et al., 2006). For each of the two datasets, we constructed 48 models using the 305 lme4 package in R (Bates et al., 2016). We used a binomial distribution with a Logit link 306 function to examine the effect of social network metrics, attributes, and their potential 307 interactions on Shigella infection across individual macaques (McCullagh \& Nelder, 1989), 308 Given the potential collinearity or non-independence among centrality network metrics (Farine $309 \&$ Whitehead, 2015; Krause et al., 2007; MacIntosh et al., 2012; Sueur et al., 2011a), we 310 refrained from using the automated 'Dredge' function that provides a full set of sub-models for a 311 set of predictors (Grueber et al., 2011). Instead, our complete model-set (48 models for each 312 dataset: Supporting Table 2, 3) was composed of individually-constructed models, specifically a 313 'null' model (1 model), a model each for the main effect of each of nine network metrics (11 314 models), combinations of two uncorrelated network metrics (grooming or huddling with 315 aggression) to examine their combined main effects (28 models), main effects plus a sex $\times$ 316 dominance rank interaction (1 model), and main effects plus an interaction between each groom 317 or huddle centrality metric and dominance certainty (7 models). From these, we selected our 318 candidate model-set by selecting all models with a dAICc (or $\Delta \mathrm{AIC})<2$ (Burnham et al., 2011), 319 and using the law of parsimony to eliminate models whose increased complexity does not 320 improve AICc over a simpler model in the candidate set (Burnham et al., 2011; Grueber et al.,

321 2011; Richards, 2005, 2008). We report coefficients and other summary statistics $(\mathrm{P}=0.05$ as 322 'significant', $0.5<\mathrm{P}<0.1$ as a 'nonsignificant trend') from each model within our candidate 323 model-sets.

\section{Results}


327 detected a moderate-low prevalence of Shigella among the CNPRC macaques. Specifically,

328 Group I had the highest prevalence, with 23 out of 101 individuals being infected ( 23\%).

329 Groups II and III showed similarly low prevalence, i.e. 8 out of $96(8.33 \%)$ and 7 out of 100

$330(7 \%)$ sampled individuals respectively.

Infection, Network Metrics, and Individual Attributes: In groups I and II, we found that well-connected individuals were socially buffered against the risk of Shigella infection. There were four models in the candidate model-set for the analysis of groups I and II (Table 1). These revealed significant, negative relationships between Shigella infection and three network metrics -- groom out-degree (Model 4), groom eigenvector centrality (Model 7; Figure 2), and huddle betweenness (Model 9) (Table 1). In other words, infection was least likely among individuals with strongest grooming and huddling connections. Models other than the candidate models also showed negative relationships between grooming and huddling connectedness and infection (e.g. groom betweenness: Model 5; huddle degree: Model 7; Supporting Table 2). Further, there was a significant interaction between huddling betweenness and dominance certainty (Model 20).

341 When explored further, we found that individuals with categorically low certainties (below the $34250^{\text {th }}$ percentile) showed strong, negative relationships between huddling betweenness and 343 infection $(\beta=-4.19, p=0.02)$ whereas those with categorically high dominance certainties 344 showed no such effects $(\beta=0.38, p=0.82)$. In other words, social buffering via huddling 345 connections was more discernible among individuals with low dominance certainties than those 346 with high dominance certainties. Finally, models that included aggression network metrics 347 and/or sex interacting with dominance rank were not part of the candidate model set (Table 1;

348 Supporting Table 2, 3), suggesting no clear relationships between these variables and infection. 

were involved in more aggressive interactions and/or more central in their huddling networks, suggesting contact-mediated transmission. Specifically, the candidate model-set constituted four models (Table 2) that revealed significant, positive relationships between Shigella infection and 353 both aggression out-degree (Models 42, 11; Figure 3, 4) and out-strength (Models 41, 13).

354 Models other than the candidate models also showed positive associations between possessing

355 both direct and indirect grooming and huddling connections and Shigella infection (e.g. groom 356 indegree: Model 4; huddle degree: Model 7; Huddle eigenvector: Model 9; Supporting Table 3).

357 There was also a non-significant positive trend between infection risk and huddle betweenness

358 (Models 41, 42) (Table 2; Figure 4). When we examined metrics from aggression networks with 359 only intense aggressive interactions (bites, attacks, i.e. those involving contact), the positive 360 relationships were sustained but nonsignificant (Model 42: contact-aggression out-degree: $\beta=$ $3613.37, p=0.07$; huddle betweenness: $\beta=2.87, p=0.08$ ). Finally, models that included grooming 362 network metrics and/or sex interacting with dominance rank were not part of the candidate model 363 set (Table 2; Supporting Table 2, 3), suggesting no clear relationships with infection.

\section{Discussion}

In animal societies, the way in which sociality impacts individual health and fitness

367 remains hotly debated. In regards to infectious disease susceptibility, increased social

368 connections among group members may either facilitate the acquisition and transmission of 369 pathogens via social contact (Drewe, 2010b; Drewe \& Perkins, 2015; Freeland, 1976; Loehle, 370 1995; MacIntosh et al., 2012), or may inhibit such acquisition via socially buffering individuals 371 against daily stressors to reduce the risk of environmental acquisition of pathogens (Cohen et al., 
372 2015; Hennessy et al., 2009; Kaplan et al., 1991; Young et al., 2014). Results from our study

373 speak to both of these processes. Specifically, they reveal that among two groups of rhesus

374 macaques (a species characterized by strong social bonds (Sade, 1972)), having greater social

375 connections in both grooming and huddling social networks socially buffered individuals against

376 the risk of infection from an enteric pathogen, Shigella flexneri. Yet in a third group, we found

377 that individuals who gave more aggression and/or functioned as centralized links or hubs of

378 information flow between other individuals, were more likely to be infected, supporting the 379 contact-mediated transmission hypothesis.

380 Nonhuman primates harbor a variety of pathogens, several of which also infect humans

381 (Engel \& Jones-Engel, 2011; Jones-Engel et al., 2005; Kaur \& Singh, 2009; Nunn, 2012; Nunn

382 \& Altizer, 2006). In comparison with viruses and ectoparasites, the prevalence of zoonotic

383 bacteria are relatively understudied (Nunn \& Altizer, 2006). We found a moderate-to-low

384 prevalence of Shigella flexneri in captive rhesus macaques, which is consistent with previous

385 studies of bacterial prevalence in other free-living primate populations (e.g. free-living rhesus

386 macaques: (Beisner et al., 2016), savannah baboons (Papio anubis: (Drewe et al., 2012)). Such

387 comparisons reflect the general dearth of studies on bacterial prevalence among captive animals;

388 on which studies to date have mostly focused on the clinical bases, i.e. the virulent versus

389 asymptomatic effects, of infection among individuals (Lee et al., 2011; Shipley et al., 2010).

390 Indeed, the last documented reports of group- or population-specific prevalence levels of enteric

391 bacteria among captive primates were on rhesus (23\% of 4476 individuals: (Good et al., 1969))

392 and longtailed macaques (18.8\% of 1297 individuals: (Takasaka et al., 1964)) imported into

393 biomedical facilities during the 60s. Given the now well-established, reliable characterization

394 approaches (see Methods), our reports of low prevalence were not likely artifacts of 
395 methodological issues. Among humans on the other hand, reports of enteric bacterial prevalence

396 are often inflated by sampling paradigms that focus purely on hospitalized patients, and/or

397 individuals already showing signs of symptoms (e.g. (Kotloff et al., 1999)). We contend that pre-

398 symptomatic, epidemiologically accurate assessments of enteric bacterial prevalence levels are

399 imperative both for humans and captively housed animals given that these pathogens are (i)

400 omnipresent, (ii) both socially and environmentally transmittable, and/or (iii) may cause

401 unpredictable outbreaks of virulent infection both among humans and macaques (DuPont, 2000;

402 Gupta et al., 2004; Kessler \& Rawlins, 2016). Among the CNPRC macaques, a logical next step

403 would be to establish associations between Shigella infection and symptomatic effects (e.g.

404 diarrhea, enteritis) that may necessitate clinical treatment.

405 Animal studies that demonstrate the impact of social buffering on health and stress

406 mitigation have traditionally focused on stress-related health outcomes in pair-housed or

407 monogamous species (reviewed in (Hennessy et al., 2009)), with evidence among socially

408 cohesive, group-living species with strong social bonds emerging only more recently (e.g.

409 Chacma baboons (Papio hamadryas ursinus): (Archie et al., 2014; Silk et al., 2010); Barbary

410 macaques (M. sylvanus: (Young et al., 2014); humans: (Holt-Lunstad et al., 2010)). Our findings

411 extend the impact of social buffering to infectious agent acquisition. Among two groups of

412 rhesus macaques, our candidate models revealed that individuals possessing direct and secondary

413 grooming connections, and strong huddling connections particularly among individuals with

414 uncertain dominance relationships, were the least prone to infection from Shigella flexneri.

415 Broadly, these findings add novelty to epidemiological studies implementing social networks by

416 contradicting the popularly prevailing notion that infectious agent acquisition is a consistent

417 drawback of group living (Alexander, 1974; Freeland, 1976; Loehle, 1995; MacIntosh et al., 
418 2012). The negative relationship between Shigella infection risk and each of grooming outdegree

419 and eigenvector centrality points to grooming being a strong source of social buffering. This is

420 consistent with prior research among primates that has revealed that both giving and receiving

421 grooming may mitigate social stress (Henzi \& Barrett, 1999; Smutt et al., 2007). Further, the

422 establishment of the social buffering phenomenon in a second type of affiliation network

423 (huddling) improves on most epidemiological studies that focus on just one (reviewed in (Drewe $424 \&$ Perkins, 2015)).

425 Specifically, the effect of grooming outdegree suggests that giving grooming may be

426 more beneficial in lowering infection risk than receiving grooming, via potentially functioning to

427 elicit additional buffering-related benefits such as social support (Seyfarth, 1977; Smutt et al.,

428 2007). This argument is further supported by the effect of grooming eigenvector centrality, i.e.

429 having a well-connected social circle of primary and secondary connections to elicit such

430 support (Farine \& Whitehead, 2015), on also lowering infection risk. Surprisingly, eigenvector

431 centrality has been frequently overlooked in infectious disease research in favor of metrics based

432 on direct connections (Drewe \& Perkins, 2015). Yet one study (MacIntosh et al., 2012) found

433 that grooming eigenvector centrality increased likelihood of infection from a nematode parasite

434 (Strongyloides fuelleborni) in wild Japanese macaques, suggesting contact-mediated

435 transmission. Our finding revealed an opposite, social buffering-mediated effect. Such an effect

436 may be particularly discernible in study systems, such as large groups of captive macaques, that

437 are socially (in addition to biologically: (Suomi, 2011)) analogous to dense human communities

438 in urban settings wherein having a broad circle of social connections also has well-documented

439 health- and fitness-related benefits (Janowski et al., 2012; Uchino, 2004, 2009). 
441 also more socially buffered against infection. Where social buffering prevails, betweenness,

442 which measures the relative number of pathways that pass through each individual, may be a

443 more reliable indicator of the strength and extent of support ties in networks (such as huddling)

444 where the direction of the relationship (unlike in grooming) is less significant. Further, the effect

445 of huddling betweenness in mitigating infection risk was particularly strong among individuals

446 with more uncertain dominance relationships. Such a complex relationship between dominance

447 status, network connectedness, and infection risk is consistent with what we see in the literature.

448 The effect of dominance status on infection risk varies by social system and the type of health

449 outcome examined. In primate groups for instance, high social rank is related to greater access to

450 grooming partners and may enhance contact-associated susceptibility to infectious diseases

451 (MacIntosh et al., 2012). Further, the relationship between dominance rank and stress (a proxy

452 for infectious disease susceptibility via social buffering (Capitanio \& Cole, 2015)) is highly

453 inconsistent, with both high- and low-ranking individuals experiencing different types of social

454 and biological stressors (e.g. (Abbott et al., 2003; Sapolsky, 2005), but see (Foerster et al.,

455 2015)). Thus dominance status, rather than linearly predicting infection risk, may instead interact

456 with stress-levels and/or social network connectedness to influence this risk. To assess

457 dominance status, we use the measure of certainty in addition to rank, which had no effects.

458 Dominance certainty differs from rank; it is a metric of the predictability of the direction of an

459 individual's dominance interactions and pathways of interactions, irrespective of the outcome of

460 wins or losses (Fujii et al., 2014). Biologically, it may be a more important moderator (than rank)

461 on health outcomes. For instance, a recent study on the CNPRC rhesus macaques showed that

462 individuals that face greater unpredictability in their dominance encounters also showed 
463 pronounced biomarkers of poor health, including inflammatory proteins and diarrhea

464 (Vandeleest et al., 2016). Under such circumstances, the beneficial impact of possessing strong

465 social connections in buffering individuals against infection risk may be more clearly

466 discernible. A clearer picture on the effects of dominance on infection risk may emerge when

467 future studies examine the effects of dominance certainty instead of or in addition to rank.

$468 \quad$ Our findings should lead to future investigations of whether increased network

469 connectedness mitigates biological stress indicators (e.g. Glucocorticoid or GC content:

470 (Sapolsky et al., 2000; Young et al., 2014)) and indeed, other systemic inflammation markers

471 (e.g. C-reactive protein (CRP), Interleukin-6 (IL-6): (Libby et al., 2002)) that may influence

472 buffering-mediated infection risk, which are currently under analysis. It is also conceivable that

473 social connections may impact susceptibility versus resistance to bacterial infection via altering

474 individuals' foraging regimes and thereby modifying their gut microbial flora (Degnan et al.,

475 2012; McCord et al., 2014). Among captive long-tailed macaques (M. fascicularis), for instance,

476 commensal gut E. coli competitively inhibited infection from Shigella (Seekatz et al., 2013).

477 Thus, assessing the links between social networks and stress- and/or microbiome-mediated

478 infection risk would be logical next steps.

479 Social networks have proved to be highly beneficial in detecting contact-mediated

480 infection and transmission of pathogens (Drewe \& Perkins, 2015). Consistent with these

481 previous efforts, we found that giving more aggression to others and having more huddling

482 connections both increased individuals' risk of Shigella infection in our third rhesus macaque

483 group. Aggression may increase the risk of infection either via contact-risk (Drewe, 2010a,

484 2010b), or via weakening the health-related benefits of social buffering (Muller \& Wrangham,

485 2004; Ostner et al., 2008). The fact that aggression given, but not received, was related to 
486 infection risk better supports the former compared to the latter. Further, the positive association

487 between huddling betweenness and infection risk suggests that individuals with increased

488 huddling connections may be superspreaders of pathogens (Lloyd-Smith et al., 2005). This is

489 because when contact-transmission is prevalent, betweenness, in its calculation of the relative

490 number of pathways that pass through a node, specifically measures the potential for the flow of

491 information (or pathogens) through a node (Drewe \& Perkins, 2015; Farine \& Whitehead, 2015;

492 Newman, 2005; VanderWaal et al., 2014). It may therefore be an especially useful metric to

493 parse out 'informative' nodes among captive groups where individuals tend to come into contact

494 with more individuals than those in free-living groups, which are less spatially constrained

495 (Drewe \& Perkins, 2015; Griffin \& Nunn, 2012). Finally, our findings yielded no information on

496 Shigella transmission through Group III's grooming network. This might be because grooming

497 involves a more subtle form of hand-to-body-hair contact compared to huddling, which involves

498 more direct and prolonged body-to-body contact that may increase the chances of transmission.

499 Although contact-mediated transmission in the aggression network might be expected to

500 be most pronounced in a network composed of only intense contact-aggression, we found the

501 reverse: a weaker association. One explanation for this could be that the detectability of

502 transmission may be influenced by pathogen-specific differences in modes of transmission. For

503 instance, the transmission of viral pathogens from macaques to humans would require the

504 occurrence of specifically intense forms of contact, such as bites and scratches, which may

505 culminate in the contact-exchange of body-fluids such as blood and saliva (Engel \& Jones-Engel,

506 2011; Engel et al., 2013). Yet simpler body-contact and/or the sharing or consumption of

507 contaminated food or water source has been shown to be sufficient for the transmission of enteric

508 bacterial pathogens (like Shigella) among humans (Benjamin et al., 2013; Cooley et al., 2007). 
510 versus contact-mediated transmission of infectious agents may prevail. To our knowledge, most

511 epidemiological studies that have reported contact-mediated transmission of infectious agents

512 through social networks have focused on free-living or wild animal groups (reviewed in (Drewe

513 \& Perkins, 2015)). It is conceivable that social buffering may be more readily discernible among

514 large, spatially constrained primate groups living in captivity. As in dense, suburban populations

515 of humans (e.g. (Janowski et al., 2012; Uchino, 2004, 2009); see above), such living conditions

516 manifest in more by-standing to witness (De Marco et al., 2010), and/or less opportunities for the

517 social avoidance of, agonistic encounters (Fujii et al., 2014; McCowan et al., 2008). This may

518 amplify the utility of strong social ties as avenues of support or stress-relief.

519 Yet by itself, living condition does not explain the heterogeneous pattern observed in

520 Group III, which showed evidence for contact-mediated transmission. We offer two potential

521 explanations for this. First, unlike Groups I and II which had diverse age-structures ( $3-22$ year

522 olds), Group III was primarily composed of younger individuals (3 - 11 years of age)

523 (Supporting Table 1). Among free-ranging rhesus macaques, age proximity has been shown to

524 positively influence the quality of affiliative social relationships (Widdig et al., 2001). Similarly,

525 it may be likely that social ties among younger animals, on account of being more nascent and/or

526 unpredictable, are not of the relationship quality that may be required for social buffering.

527 Second, the discernibility of social buffering versus contact-transmission may be governed by

528 higher-order social contexts, such as group stability. For instance, both Groups I and II

529 maintained consistent dominance relationships with few reversals, as evidenced by consistencies

530 in the overall directions of dominance encounters across their aggression and submissive status

531 networks (Chan et al., 2013). In Group III, a comparison of these networks revealed marked 
532 inconsistencies in the direction of the relationships, which persisted until the group suffered a

533 social collapse around 13 weeks after the data collection period (Chan et al., 2013). Thus it is

534 conceivable that in captivity, contact-mediated transmission is more easily decipherable under

535 socially unstable conditions where the effect of social buffering is minimal or absent. Validations

536 of both these explanations await future assessments of (i) the age-classes of immediate

537 connections, or "neighborhoods", of older versus younger individuals, and (ii) expansions of

538 current findings to additional groups.

539 In summary, this study suggests that within captive housed groups of a nonhuman

540 primate biologically and socially analogous to humans, individuals' network connections may

541 socially buffer them against, or promote the contact-mediated transmission of infection from an

542 enteric bacterial pathogen. The generality of these findings awaits expansions of similar

543 approaches to additional social and pathogenic taxa. Such efforts may facilitate delineating what

544 may be a fine line between when/ how social network connections may be beneficial versus

545 detrimental to infectious disease risk.

546

\section{Acknowledgments}

548 We would like to thank our dedicated team from the McCowan Animal Behavior Laboratory,

549 including A. Nathman, A. Barnard, T. Boussina, A. Vitale, E. Cano, J. Greco, N. Sharpe, and S.

550 Seil, who participated in the behavioral data collection. We would also like to thank members of

551 the Atwill and WIFSS (Western Institute for Food Safety and Security) laboratories, particularly

552 C. Bonilla, J. Carabez, R. Pisano, and I. Wong, for playing key roles in the processing of fecal

553 samples for pathogen isolation and characterization at the School of Veterinary Medicine, UC

554 Davis. Finally, we are grateful to Dr. Hsieh Fushing and his research team at the Department of 
555 Statistics for the development and Percolation-and-Conductance method. We also thank him and

556 his team along with Jian Jin for guidance in the implementation of the Percolation-and-

557 Conductance (Perc) R package.

558

559

560

561

562

563

564

565

566

567

568

569

570

571

572

573

574

575

576

577

578

579

580

581

582

583

584

585

586

587

588

589

590

591

592

593

594

\section{References}

Abbott, D. H., Keverne, E. B., Bercovitch, F. B., Shively, C. A., Mendoza, S. P., Saltzman, W., Snowdon, C. T., Ziegler, T. E., Banjevic, M., Garland, T. J., \& Sapolsky, R. M. (2003). Are subordinates always stressed? A comparative analysis of rank differences in cortisol levels among primates. Horm. Behav., 43, 67-82.

Alexander, R. D. (1974). The evolution of social behaviour. Annu. Rev. Ecol. Syst., 5, 325-383.

Altmann, J. (1974). Observational study of behavior: sampling methods. Behaviour, 49, 227266.

Anderson, R. M., \& May, R. M. (1992). Infectious diseases of humans: dynamics and control. Oxford: Oxford University Press.

Archie, E. A., Tung, J., Clark, J., Altmann, J., \& Alberts, S. C. (2014). Social affiliation matters: both same-sex and opposite-sex relationships predict survival in wild female baboons. Proc Biol Sci, 281, 1973.

Bansal, S., Grenfell, B. T., \& Meyers, L. A. (2007). When individual behaviour matters: homogeneous and network models in epidemiology. J Royal Soc Interface, 4, 879891.

Bates, D., Maechler, M., Bolker, B., Walker, S., Christensen, R. H. B., Singmann, H., Dai, B., Grothendieck, G., \& Green, P. (2016). Linear mixed-effects models using 'eigen' and S4. Retrieved from https://github.com/lme4/lme4/ http://lme4.r-forge.rproject.org/

Beisner, B. A., Balasubramaniam, K. N., Fernandez, K., Heagerty, A., Seil, S. K., Atwill, E. R., Gupta, B. K., Tyagi, P. C., Chauhan, N. P. S., Bonal, B. S., Sinha, P. R., \& McCowan, B. (2016). Prevalence of enteric bacterial parasites with respect to anthropogenic factors among commensal rhesus macaques in Dehradun, India. Primates, Published online:, http://dx.doi.org/10.1007/s10329-10016-10534-10322

Benjamin, L., Atwill, E. R., Jay-Russell, M. T., Cooley, M., Carychao, D., Gorski, L., \& Mandrell, R. E. (2013). Occurrence of generic E. coli, E. coli 0157:H7 and Salmonella spp. in water and sediment from leafy green produce farms and streams on the Central California coast. International Journal of Food Microbiology, 165, 65-76. doi: http://dx.doi.org/10.1016/j.ijfoodmicro.2013.04.003

Berman, C. M., \& Thierry, B. (2010). Variation in kin bias: species differences and time constraints in macaques. 147(13), 1863-1887. doi: doi:10.1163/000579510X539691

Burnham, K. P., Anderson, D. R., \& Huyvaert, K. P. (2011). AIC model selection and multimodel inference in behavioral ecology: some background, observations, and comparisons. Behav. Ecol. Sociobiol., 65, 23-35. 
595 Capitanio, J., \& Cole, S. W. (2015). Social instability and immunity in rhesus monkeys: the role of the sympathetic nervous system. Phil. Trans. R. Soc. B, 370, 20140104.

601

602

603

604

605

606

607

608

609

610

611

612

613

614

Chan, S., Fushing, H., Beisner, B. A., \& McCowan, B. (2013). Joint Modeling of Multiple Social Networks to Elucidate Primate Social Dynamics: I. Maximum Entropy Principle and Network-Based Interactions. Plos One, 8(2). doi: 10.1371/journal.pone.0051903

Chapais, B. (2006). Kinship, competence and cooperation in primates In P. M. Kappeler \& C. P. Van Schaik (Eds.), Cooperation in Primates and Humans: An Evolutionary Perspective (pp. 47-64). Berlin: Springer-Verlag.

Cobb, S. (1976). Social support as a moderator of life stress. Psychosom Med, 38, 300-314.

Cohen, S., Janicki-Deverts, D., \& Miller, G. E. (2007). Psychological stress and disease. JAMA, 298, 1685-1687.

Cohen, S., Janicki-Deverts, D., Turner, R. B., \& Doyle, W. J. (2015). Does hugging provide stress-buffering social support? A study of susceptibility to upper respiratory infection and illness. Psychological Science, 26, 135-147.

Cooley, M., Carychao, D., Crawford-Miksza, L., Jay, M. T., Myers, C., Rose, C., Keys, C., Farrar, J., \& Mandrell, R. E. (2007). Incidence and Tracking of Escherichia coli 0157:H7 in a Major Produce Production Region in California. Plos One, 2(11). doi: 10.1371/journal.pone.0001159

De Marco, A., Cozzolino, R., Dessi-Fulgheri, F., \& Thierry, B. (2010). Conflicts induce affiliative interactions among bystanders in a tolerant species of macaque (Macaca tonkeana). Animal Behaviour, 80, 197-203.

Degnan, P. H., Pusey, A. E., Lonsdorf, E. V., Goodall, J., Wroblewski, E. E., Wilson, M. L., Rudicell, R. S., Hahn, B. H., \& Ochman, H. (2012). Factors associated with the diversification of the gut microbial communities within chimpanzees from Gombe National Park. Proceedings of the National Academy of Sciences of the United States of America, 109(32), 13034-13039. doi: 10.1073/pnas.1110994109

Drewe, J. A. (2010a). Who infects whom? Social networks and disease transmission in wild meerkats. Proc Biol Sci, 277, 633-642.

Drewe, J. A. (2010b). Who infects whom? Social networks and tuberculosis transmission in wild meerkats. Proc Biol Sci, 277, 633-642.

Drewe, J. A., O'Riain, M. J., Beamish, E., Currie, H., \& Parsons, S. (2012). Survey of infections transmissible between baboons and humans, Cape Town, South Africa. Emerging Infectious Disease, 18, 298-301.

Drewe, J. A., \& Perkins, S. E. (2015). Disease transmission in animal social networks. In J. Krause, R. James, D. W. Franks \& D. P. Croft (Eds.), Animal Social Networks (pp. 95110). Oxford: Oxford University Press.

DuPont, H. L. (2000). Shigella species (bacillary dysentery). In G. L. Mandell, J. E. Bennett \& R. Dolin (Eds.), Mandell, Douglas and Bennett's Principles and Practice of Infectious Diseases, 5th Edition (pp. 2363-2369).

Engel, G., \& Jones-Engel, L. (2011). The role of Macaca fascicularis in infectious disease transmission. In M. D. Gumert, A. Fuentes \& L. Jones-Engel (Eds.), Monkeys on the Edge: Ecology and Management of Long-tailed Macaques and their Interface with Humans (pp. 183-203). Cambridge: Cambridge University Press.

Engel, G., Small, C. T., Soliven, K., Feeroz, M. M., Wang, X., Hasan, M. K., Oh, G., Alam, S. M. R., Craig, K. L., Jackson, D. L., Matsen, F. A., Linial, M. L., \& Jones-Engel, L. (2013). Zoonotic simian foamy virus in Bangladesh reflects diverse patterns of transmission 
641

642

643

644

645

646

647

648

649

650

651

652

653

654

655

656

657

658

659

660

661

662

663

664

665

666

667

668

669

670

671

672

673

674

675

676

677

678

679

680

681

682

683

684

and co-infection. Emerging Microbes \& Infections, 2, e58. doi: doi:10.1038/emi.2013.60

Farine, D. R., \& Whitehead, H. (2015). Constructing, conducting and interpreting animal social network analysis. Journal of Animal Ecology, 84, 1144-1163.

Foerster, S., Kithome, K., Cords, M., \& Monfort, S. L. (2015). Social status and helminth infections in female forest guenons (Cercopithecus mitis). American Journal of Physical Anthropology, 158, 55-66.

Freeland, W. J. (1976). Pathogens and the evolution of primate sociality. Biotropica, 8, 1224.

Fujii, K., Fushing, H., Beisner, B. A., \& McCowan, B. (2014). Computing power structures in directed biosocial networks: flow percolation and imputed conductance. Plos One, submitted.

Godfrey, S. S., Bull, C. M., James, R., \& Murray, K. (2009). Network structure and parasite transmission in a group-living lizard, the gidgee skink, Egernia stokesii. Behav. Ecol. Sociobiol., 63, 1045-1056.

Good, R. C., May, B. D., \& Kawatomari, T. (1969). Enteric pathogens in monkeys. J. Bacteriol., 97, 1048-1055.

Griffin, R. H., \& Nunn, C. L. (2012). Community structure and the spread of infectious disease in primate social networks. Evolutionary Ecology, 26(4), 779-800. doi: 10.1007/s10682-011-9526-2

Grueber, C. E., Nakagawa, S., Laws, R. J., \& Jamieson, I. G. (2011). Multimodel inference in ecology and evolution: challenges and solutions. Journal of Evolutionary Biology, 24, 699-711.

Gupta, A., Polyak, C. S., Bishop, R. D., Sobel, J., \& Mintz, E. D. (2004). Laboratory-confirmed Shigellosis in the United States, 1989-2002: Epidemiologic Trends and Patterns. Clinical Infectious Diseases, 38, 1372-1377.

Hamede, R. K., Bashford, J., McCallum, H., \& Jones, M. (2009). Contact networks in a wild Tasmanian devil (Sarcophilus harrisii) population: using social network analysis to reveal seasonal variability in social behaviour and its implications for transmission of devil facial tumour disease. Ecology Letters, 12(11), 1147-1157. doi: 10.1111/j.1461-0248.2009.01370.x

Handcock, M., Hunter, D., Butts, C., Goodreau, S., \& Morri`s, M. (2006). Statnet: An R package for the statistical analysis and simulation of social networks. University of Washington: http://www.csde.washington.edu/statnet.

Harper, K. N., Fyumagwa, R. D., Hoare, R., Wambura, P. N., Coppenhaver, D. H., Sapolsky, R. M., Alberts, S. C., Tung, J., Rogers, J., \& Kilewo, M. (2012). Treponema pallidum infection in the wild baboons of East Africa: distribution and genetic characterization of the strains responsible. Plos One, 7, e50882.

Hennessy, M. B., Kaiser, S., \& Sachser, N. (2009). Social buffering of the stress response: Diversity, mechanisms, and functions. Front Neuroendocrinol, 30, 470-482.

Henzi, S. P., \& Barrett, L. (1999). The value of grooming to female primates. Primates, 40(1), 47-59.

Holt-Lunstad, J., Smith, T. B., \& Layton, J. B. (2010). Social relationships and mortality risk: A meta-analytic review. PLoS Med, 7, e1000316. 
685

686

687

688

689

690

691

692

693

694

695

696

697

698

699

700

701

702

703

704

705

706

707

708

709

710

711

712

713

714

715

716

717

718

719

720

721

722

723

724

725

726

727

728

729

Janowski, K., Steuden, S., Pietrzak, A., Krasowska, D., Kaczmarek, L., Gradus, I., \& Chodorowska, G. (2012). Social support and adaptation to the disease in men and women with psoriasis. Arch. Dermatol. Res., 304, 421-432.

Jones-Engel, L., Engel, G. A., Schillaci, M. A., Rompis, A. L. T., Putra, A., Suaryana, K. G., Fuentes, A., Beers, B., Hicks, S., White, R., Wilson, B., \& Allan, J. S. (2005). Primate to human retroviral transmission in Asia. Emerging Infectious Disease, 7, 1028-1035.

Kaplan, J. R., Heise, E. R., Manuck, S. B., Shively, C. A., Cohen, S., Rabin, B. S., \& Kasprowicz, A. L. (1991). The relationship of agonistic and affiliative behavior patterns to cellular immune function among cynomolgus monkeys Macaca- fascicularis living in unstable social groups. American Journal of Primatology, 25(3), 157-174.

Kappeler, P. M., Cremer, S., \& Nunn, C. L. (2015). Sociality and health: impacts of sociality on disease susceptibility and transmission in animal and human societies. Phil. Trans. $R$. Soc. B, 370, 20140116.

Kaur, T., \& Singh, J. (2009). Primate-parasitic zoonoses and anthropozoonoses: a literature review. In M. A. Huffman \& C. A. Chapman (Eds.), Primate Parasite Ecology: The Dynamics and Study of Host-Parasite Relationships (pp. 199-230). Cambridge: Cambridge University Press.

Kessler, M. J., \& Rawlins, R. G. (2016). A 75-year pictorial history of the Cayo Santiago rhesus monkey colony. American Journal of Primatology, 78, 6-43.

Kikusui, T., Winslow, J. T., \& Mori, Y. (2006). Social buffering: Relief from stress and anxiety. Phios Trans R Soc Lond B Biol Sci, 361, 2215-2228.

Kimura, R. (1998). Mutual grooming and preferred associate relationships in a band of freeranging horses. Applied Animal Behaviour Science, 59, 265-276.

Kotloff, K. L., Winickoff, J. P., Ivanoff, B., Clemens, J. D., Swerdlow, D. L., Sansonetti, P. J., Adak, G. K., \& Levine, M. M. (1999). Global burden of Shigella infections: implications for vaccine development and implementation of control strategies. Bull WHO, 77, 651-666.

Krause, J., Croft, D. P., \& James, R. (2007). Social network theory in the behavioural sciences: potential applications. Behav. Ecol. Socioecol., 62, 15-27.

Lee, J., Kim, S., \& Park, C. (2011). Shigella flexneri infection in a newly acquired rhesus macaque (Macaca mulatta). Lab Anim Res., 27, 343-346. doi: 10.5625/lar.2011.27.4.343

Libby, P., Ridker, P. M., \& Maseri, A. (2002). Inflammation and Atherosclerosis. Circulation, 105(9), 1135-1143.

Lindburg, D. G. (1971). The rhesus monkey in north India: an ecological and behavioral study. In L. A. Rosenblum (Ed.), Primate Behavior: Developments in Field and Laboratory Research (pp. 1-106). New York: Academic Press.

Lloyd-Smith, J. O., Schreiber, S. J., Kopp, P. E., \& Getz, W. M. (2005). Superspreading and the effect of individual variation on disease emergence. Nature, 438(7066), 355-359. doi: $10.1038 /$ nature 04153

Loehle, C. (1995). Social barriers to pathogen transmission in wild animal populations. Ecology, 76, 326-335.

MacIntosh, A. J. J., Jacobs, A., Garcia, C., Shimizu, K., Mouri, K., Huffman, M. A., \& Hernandez, A. D. (2012). Monkeys in the middle: parasite transmission through a social network of a wild primate. Plos One, 7, e51144. 
730

731

732

733

734

735

736

737

738

739

740

741

742

743

744

745

746

747

748

749

750

751

752

753

754

755

756

757

758

759

760

761

762

763

764

765

766

767

768

769

770

771

772

773
Makagon, M. M., McCowan, B. J., \& Mench, J. A. (2012). . How can social network analysis contribute to social behavior research in applied ethology? . Applied Animal Behavior Science (Special Issue), 138, 152-161.

McCord, A. I., Chapman, C. A., Weny, G., Tumukunde, A., Hyeroba, D., Klotz, K., Koblings, A. S., Mbora, D. N. M., Cregger, M., White, B. A., Leigh, S. R., \& Goldberg, T. L. (2014). Fecal microbiomes of non-human primates in Western Uganda reveal species-specific communities largely resistant to habitat perturbation. American Journal of Primatology, 76(4), 347-354. doi: 10.1002/ajp.22238

McCowan, B., Anderson, K., Heagarty, A., \& Cameron, A. (2008). Utility of social network analysis for primate behavioral management and well-being. Applied Animal Behaviour Science, 109(2-4), 396-405. doi: 10.1016/j.applanim.2007.02.009

McCowan, B., Beisner, B., Bliss-Moreau, E., Vandeleest, J., Jin, J., Hannibal, D., \& Hsieh, F. (2016). Connections matter: social networks and lifespan health in primate translational models. Frontiers in Psychology, Apr 22;27:433. PMC4841009.

McCowan, B., Beisner, B. A., Capitanio, J. P., Jackson, M. E., Cameron, A. N., Seil, S., Atwill, E. R., \& Hsieh, F. (2011). Network stability is a balancing act of personality, power, and conflict dynamics in rhesus macaque societies. Plos One, 6(8), e22350.

McCullagh, P., \& Nelder, J. A. (1989). Generalized Linear Models. London: Chapman and Hall. Moore, D., Angel, J. E., Cheeseman, I. M., Robinson, G. E., \& Fahrbach, S. E. (1995). A highly specialized social grooming honey bee (Hymenoptera: Apidae). Journal of Insect Behavior, 8, 855-861.

Muller, M., \& Wrangham, R. (2004). Dominance, cortisol and stress in wild chimpanzees (Pan troglodytes schweinfurthii). Behav. Ecol. Sociobiol., 55, 332-340.

Newman, M. E. J. (2005). A measure of betweenness centrality based on random walks. Social Networks, 27, 39-54.

Nunn, C. L. (2012). Primate disease ecology in comparative and theoretical perspective. American Journal of Primatology, 74(6), 497-509. doi: 10.1002/ajp.21986

Nunn, C. L., \& Altizer, S. M. (2006). Infectious Diseases in Primates: Behavior, Ecology and Evolution. Oxford: Oxford University Press.

Ostner, J., Heistermann, M., \& Schulke, O. (2008). Dominance, aggression and physiological stress in wild male Assamese macaques (Macaca assamensis). Horm. Behav., 54, 613619.

Richards, S. A. (2005). Testing ecological theory using the information-theoretic approach: examples and cautionary results. Ecology, 86, 2805-2814.

Richards, S. A. (2008). Dealing with overdispersed count data in applied ecology. J. Appl. Ecol., 45, 218-227.

Rimbach, R., Bisanzio, D., Galvis, N., Link, A., Di Fiore, A., \& Gillespie, T. R. (2015). Brown spider monkeys (Ateles hybridus): a model for differentiating the role of social networks and physical contact on parasite transmission dynamics. Phil. Trans. $R$. Soc. B, 370, 20140110.

Sade, D. S. (1972). A longitudinal study of social behavior of rhesus monkeys. . New York, Aldine Atherton: 378-398. In R. Tuttle (Ed.), The Functional and Evolutionary Biology of Primates (pp. 378-398). Chicago: Aldine-Atherton.

Sapolsky, R. M. (2005). The influence of social hierarchy on primate health. 308 , 648-652. 
774

775

776

777

778

779

780

781

782

783

784

785

786

787

788

789

790

791

792

793

794

795

796

797

798

799

800

801

802

803

804

805

806

807

808

809

810

811

812

813

814

815

816

817

818

819

Sapolsky, R. M., Romero, L. M., \& Munck, A. U. (2000). How do glucocorticoids influence stress responses? Integrating permissive, suppressive, stimulatory, and preparative actions. Endocr. Rev., 21, 55-89.

Scallan, E., Hoekstra, R. M., Angulo, F. J., Tauxe, R. V., Widdowson, M. A., Roy, S. L., Johnes, J. L., \& Griffin, P. M. (2011). Foodborne illness acquired in the United States--major pathogens. Emerging Infectious Diseases, 17, 7-15.

Seekatz, A. M., Panda, A., Rasko, D. A., Toapanta, F. R., Eloe-Fadrosh, E. A., Khan, A. Q., Liu, Z., Shipley, S. T., DeTolla, L. J., Sztein, M. B., \& Fraser, C. M. (2013). Differential Response of the Cynomolgus Macaque Gut Microbiota to Shigella Infection. Plos One, 8(6). doi: 10.1371/journal.pone.0064212

Segerstrom, S. C., \& Miller, G. E. (2004). Psychological stress and the human immune system: A meta-analytic study of 30 years of inquiry. Psychol Bull, 130, 601-630.

Seyfarth, R. M. (1977). A model of social grooming among adult female monkeys. Journal of Theoretical Biology, 65, 671-698.

Shipley, S. T., Panda, A., Khan, A. Q., Kriel, E. H., Maciel Jr, M., Livio, S., Nataro, J. P., Levine, M. M., Sztein, M. B., \& DeTolla, L. J. (2010). A challenge model for Shigella dysenteriae 1 in cynomolgus monkeys (Macaca fascicularis). Comp. Med., 60, 54-61.

Silk, J. B., Beehner, J. C., Bergman, C., Crockford, A. L., Engh, L. R., Moscovice, R. M., Wittig, R. M., Seyfarth, R. M., \& Cheney, D. L. (2010). Strong and consistent social bonds enhance the longevity of female baboons. Curr. Biol., 20,1359-1361.

Smutt, K., MacLarnon, A., Heistermann, M., \& Semple, S. (2007). Grooming in Barbary macaques: better to give than to receive? Biology Letters, 3(3), 231-233.

Sueur, C., Jacobs, A., Amblard, F., Petit, O., \& King, A. J. (2011a). How can social network analysis improve the study of primate behavior? American Journal of Primatology, 73, 703-719.

Sueur, C., Petit, O., De Marco, A., Jacobs, A. T., Watanabe, K., \& Thierry, B. (2011b). A comparative network analysis of social style in macaques. Animal Behaviour, 82(4), 845-852. doi: 10.1016/j.anbehav.2011.07.020

Suomi, S. J. (2011). Risk, resilience, and gene-environment interplay in primates. J Can Acad Child Adolesc Psychiatry, 20, 289-297.

Takasaka, M., Honjo, S., Fujiwara, T., Hagiwara, T., Ogawa, H., \& Imaizumi, K. (1964). Shigellosis in cynomolgous monkeys (Macaca irus). Jpn. J. Med. Sci. Biol., 17, 259-265.

Thierry, B. (2007). Unity in diversity: lessons from macaque societies. Evolutionary Anthropology, 16, 224-238. doi: 10.1002/evan.20147

Uchino, B. (2004). Social Support and Physical Health: Understanding the Health Consequences of Relationships. New Haven, CT: Yale University Press.

Uchino, B. (2009). Understanding the links between social support and physical health: A life-span perspective with emphasis on the separability of perceived and received support. Psychological Science, 4, 236-255.

Vandeleest, J. V., Beisner, B. A., Hannibal, D., Nathman, A., Capitanio, J., Fushing, H., \& McCowan, B. (2016). Rank and dominance certainty influence multiple health outcomes in rhesus macaques (Macaca fuscata). Submitted to PLoS ONE.

VanderWaal, K. L., Atwill, E. R., Hooper, S., Buckle, K., \& McCowan, B. (2013). Network structure and prevalence of Cryptosporidium in Belding's ground squirrels. Behavioral Ecology and Sociobiology, 67(12), 1951-1959. doi: 10.1007/s00265-0131602-x 
820 VanderWaal, K. L., Atwill, E. R., Isbell, L. A., \& McCowan, B. (2014). Quantifying microbe

821

822

823

824

825

826

827

828

829

830

831

832 transmission networks for wild and domestic ungulates in Kenya. Biological Conservation, 169, 136-146. doi: 10.1016/j.biocon.2013.11.008

Whittingham, M. J., Stephens, P. A., Bradbury, R. B., \& Freckleton, R. P. (2006). Why do we still use stepwise modelling in ecology and behaviour? Journal of Animal Ecology, 75, 1182-1189.

Widdig, A., Numberg, P., Krawczak, M., Streich, W. J., \& Bercovitch, F. B. (2001). Paternal relatedness and age proximity regulate social relationships among adult female rhesus macaques. PNAS, 98, 13769-13773.

Young, C., Majolo, B., Heistermann, M., Schulke, O., \& Ostner, J. (2014). Responses to social and environmental stress are attenuated by strong male bonds in wild macaques. PNAS, 111, 18195-18200. 


\section{Figure 1 (on next page)}

Diagnostic box-plots of between-group similarities and differences in relationships between network metrics and Shigella infection

Each plot shows the mean and distribution of a network metric ( $Y$ axis) plotted for infected and uninfected individuals in each group (X Axis). Red box-plots represent comparisons for Groups I and II, and Yellow comparisons for Group III 

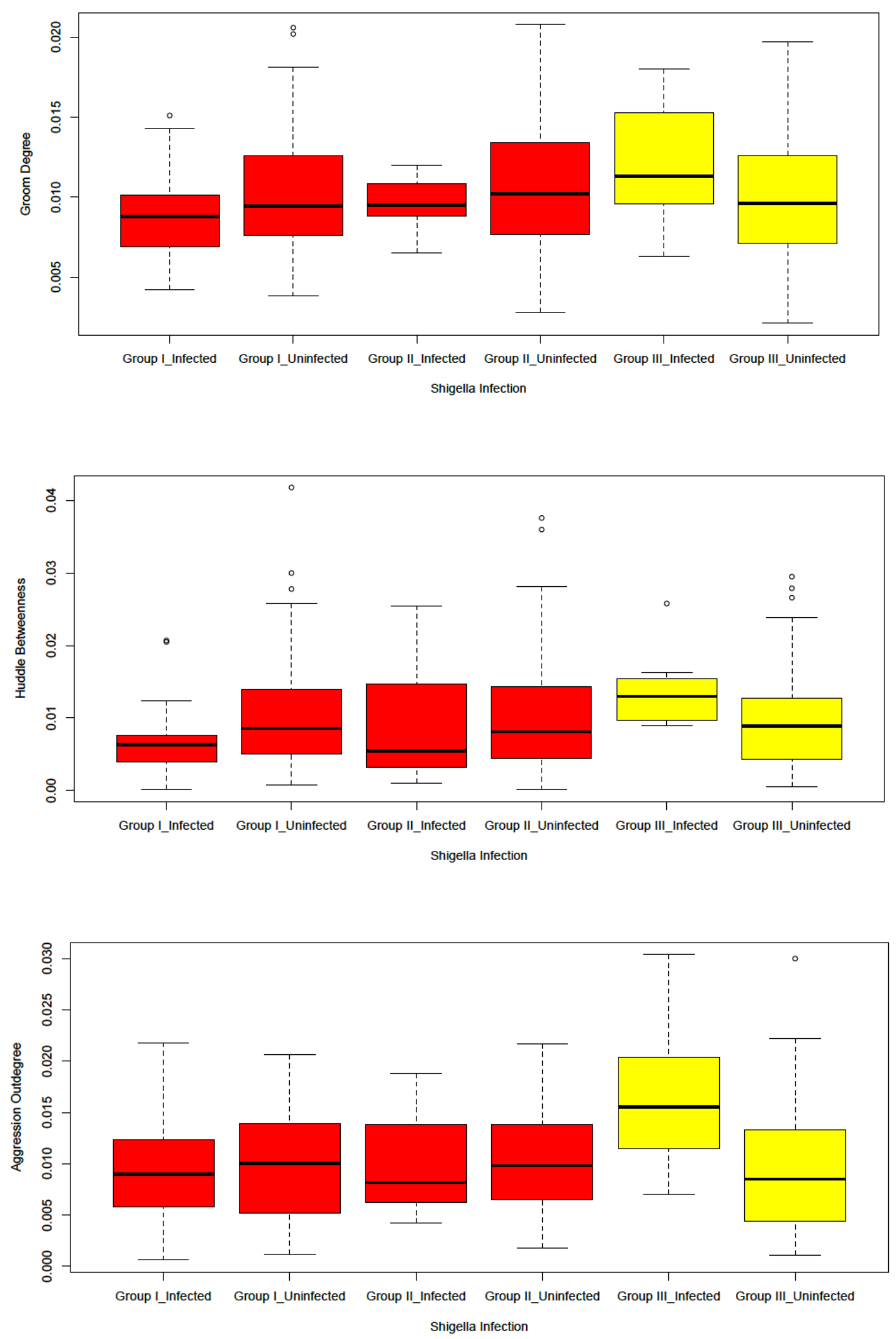


\section{Figure 2 (on next page)}

Group I: Grooming Social Network and Shigella Infection Risk

Social Network graph of grooming relationships indicating the effect of social buffering on Shigella infection in Group I. Nodes are sized proportional to the eigenvector centralites of individual macaques. Infection (yellow nodes: $N=23$ out of 101 ) is restricted to individuals with the lowest eigenvector centralities 


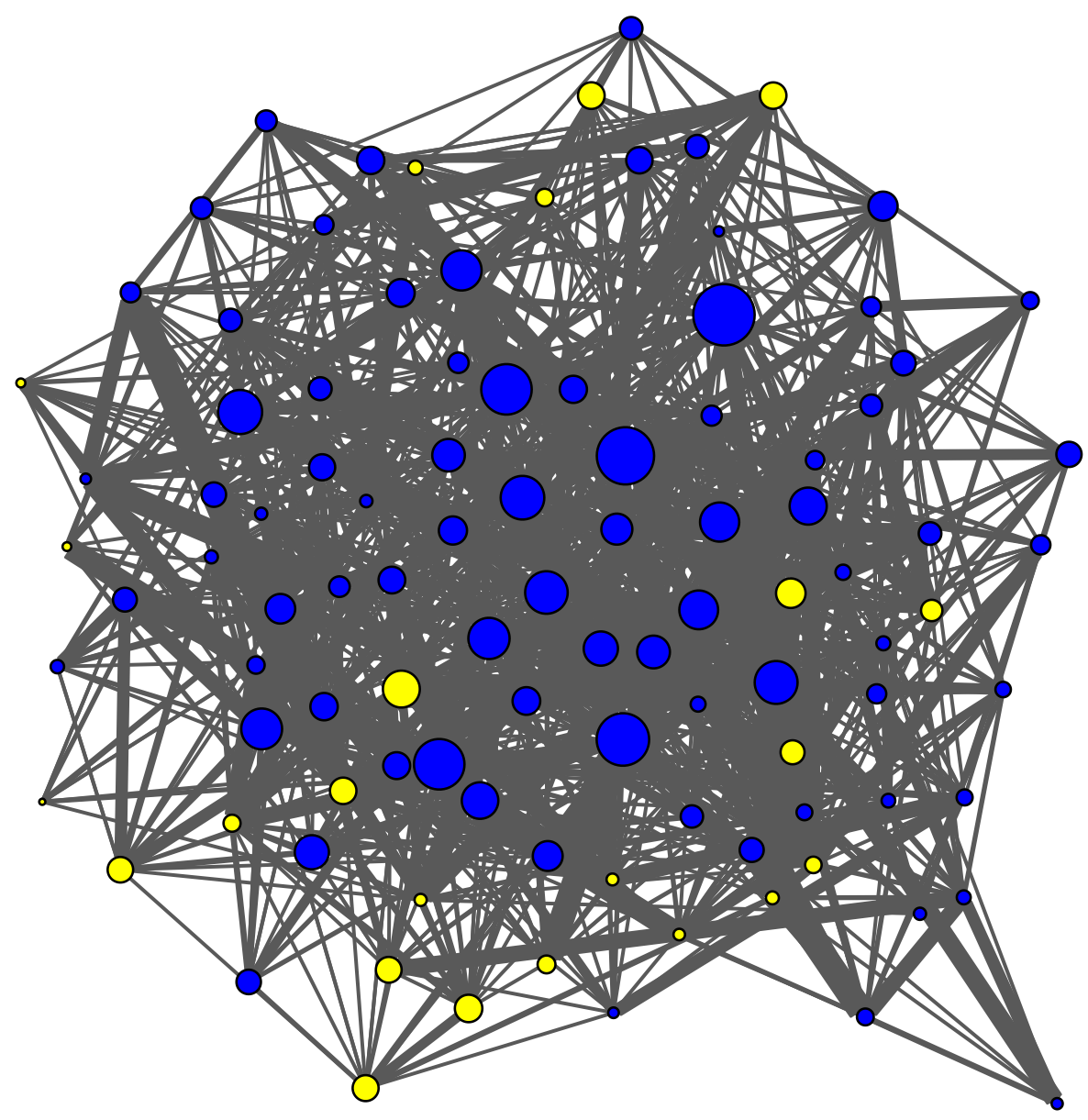




\section{Figure 3 (on next page)}

Group III: Aggression social network and Shigella infection risk

Social Network graph of aggression relationships indicating the effect of social contact on Shigella infection in Group III. Nodes are sized proportional to the aggression out-degrees of individual macaques. Infection (yellow nodes: $N=7$ out of 100 ) is prevalent among individuals with moderate-to-high out-degree 


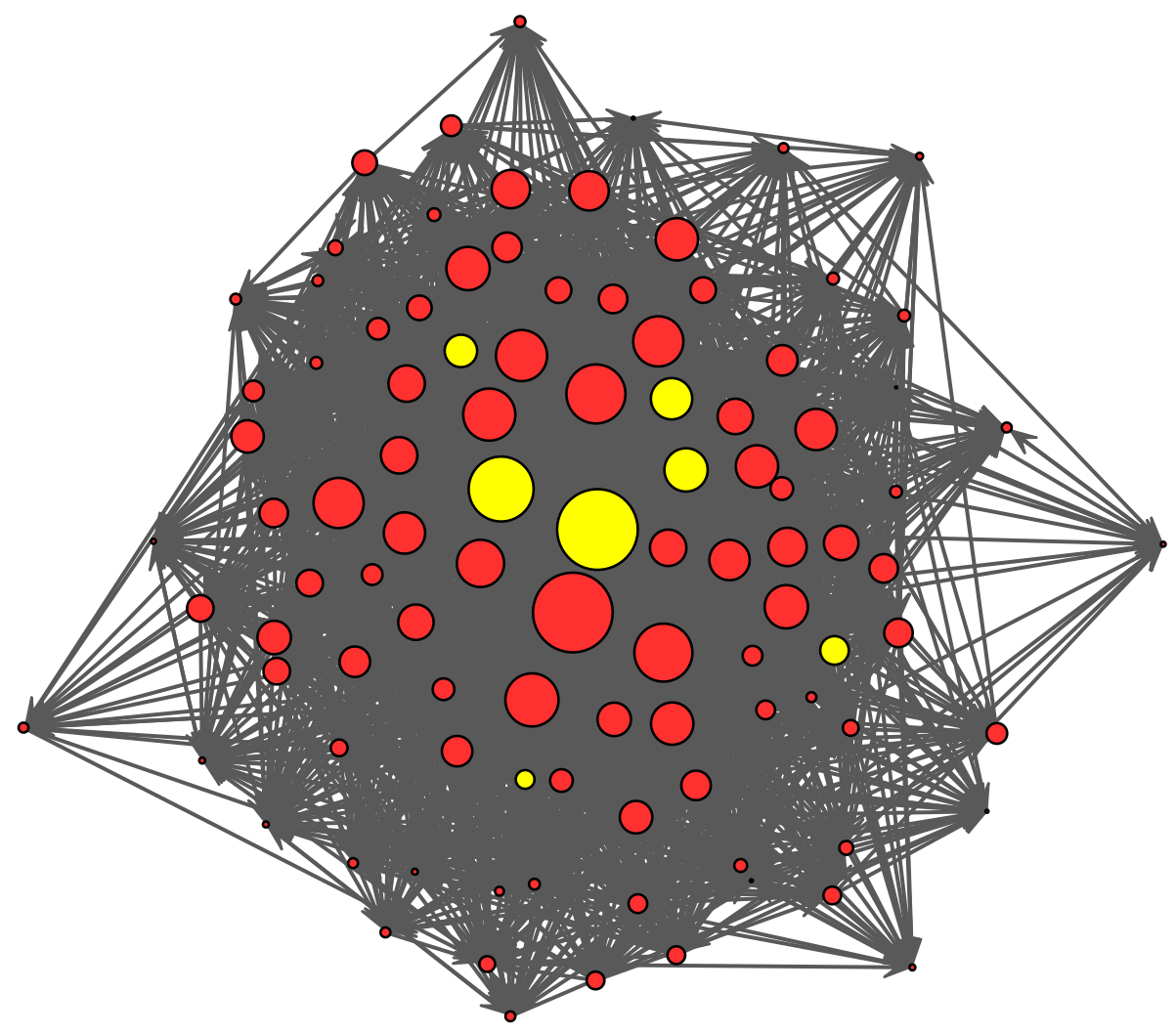




\section{Figure 4 (on next page)}

Group III: Aggression, huddling, and Shigella infection risk

Scatter-plot showing the effect of social contact on Shigella flexneri infection for Group III. Black dots are infected macaques ( $\mathrm{N}=7$ out of 100), and are concentrated among those with moderate-to-high huddle betweenness and aggression out-degree 


\section{Table $\mathbf{1}$ (on next page)}

Summary of model statistics and parameter estimates from the candidate model set (all models with a $\triangle$ AIC $<2$; outcome: Shigella presence-absence) for rhesus macaques in Groups I \& II 


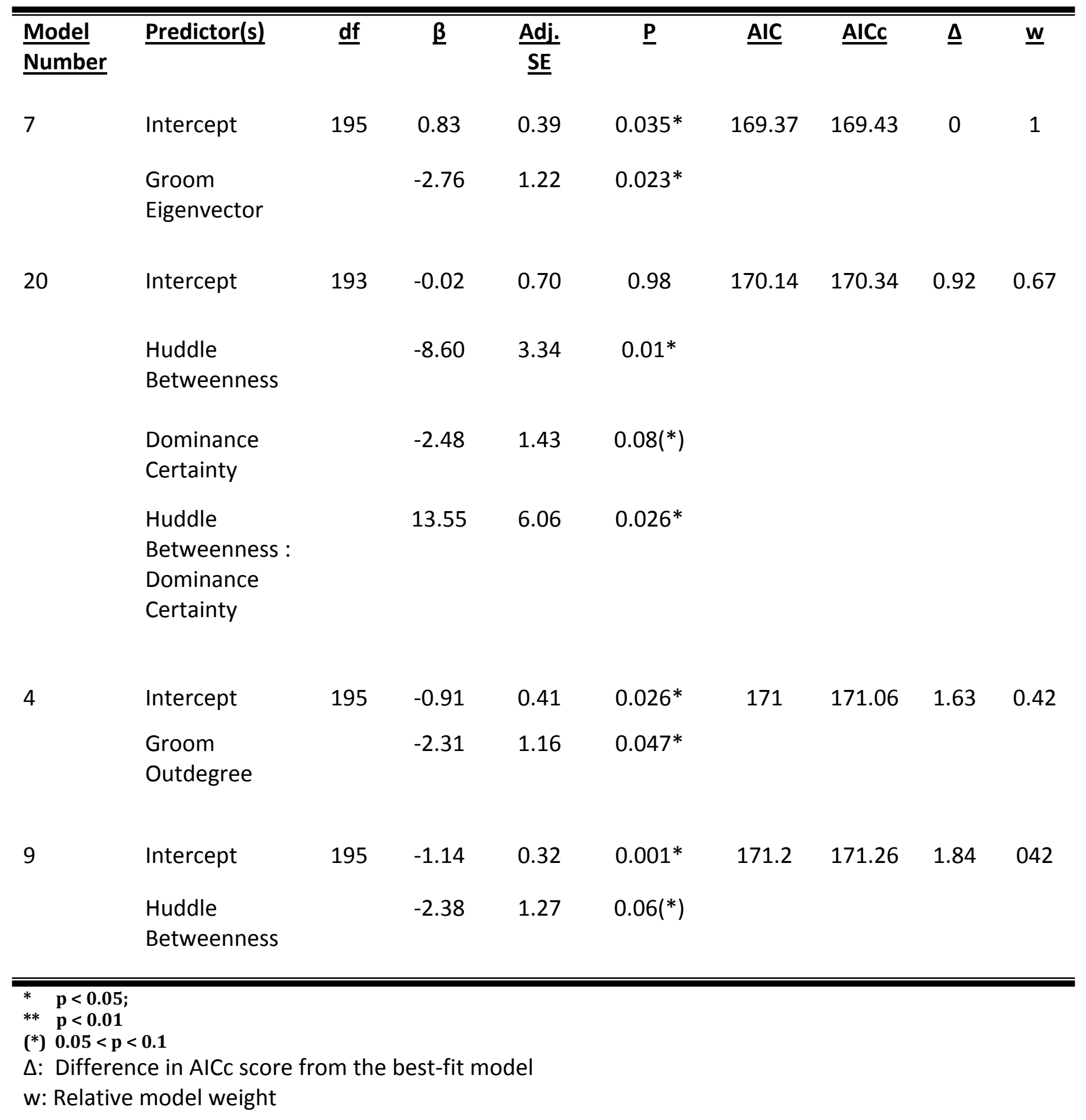




\section{Table 2 (on next page)}

Summary of model statistics and parameter estimates from the candidate model set (all models with a $\triangle$ AIC $<2$; outcome: Shigella presence-absence) for rhesus macaques in Group III 


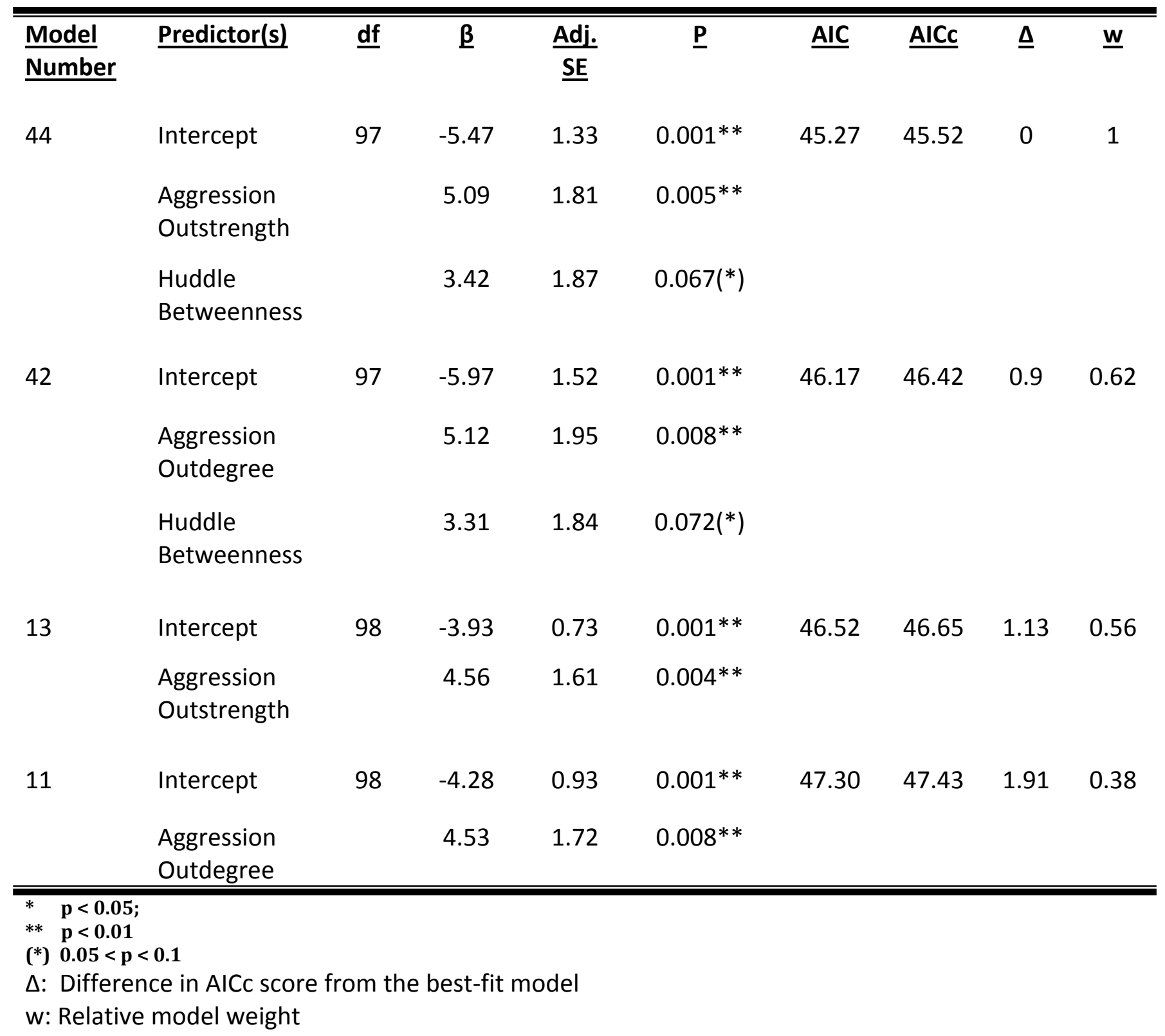

